\title{
Nano graphite platelets-enabled piezoresistive cementitious composites for structural health monitoring
}

\author{
Shengwei Sun ${ }^{1, *}$, Baoguo Han ${ }^{2, *}$, Shan Jiang ${ }^{2}$, Xun Yu³ , Yanlei Wang², \\ Hongyan $\mathrm{Li}^{2}$, Jinping $\mathrm{Ou}^{1,2}$ \\ ${ }^{1}$ School of Civil Engineering, Harbin Institute of Technology, Harbin, 150090 China \\ ${ }^{2}$ School of Civil Engineering, Dalian University of Technology, Dalian, 116024 China \\ ${ }^{3}$ Department of Mechanical Engineering, New York Institute of Technology, New York, NY 11568, USA \\ ${ }^{4}$ School of Machinery and Automation, Wuhan University of Science and Technology, Wuhan, \\ 430081 China
}

\begin{abstract}
:
This paper studied the piezoresistive effects of cementitious composites filled with nano graphite platelets (NGPs). Experiments are performed to investigate the electrical responses of cementitious composites filled with NGPs when subjected to cyclic compressive stress under different quasi static and dynamic loading conditions such as different loading amplitudes, different loading rates and constant loading within the elastic regime of these composites. Meanwhile, the cementitious composites filled with NGPs are explored for the application in measuring dynamical loading rate. The results demonstrated that cementitious composites filled with NGPs possess sensitive piezoresistive effect and stable repeatability to different loading conditions.
\end{abstract}

\footnotetext{
* Corresponding author: +8641184780087

Email address: $\underline{\text { ssw5256081@163.com, hanbaoguo@dlut.edu.cn }}$
} 
Keywords: Nano graphite platelets; cementitious composites; electrical resistivity; piezoresitivity; tunneling effect

\section{Introduction}

Conductive cementitious composite is a kind of heterogeneous material, which consists of binder, conductive material, aggregate, water, etc. Due to the good electrical conductivity and multifunctionality (e.g. joule effect, thermo-electric effect, and piezoresistive effect), the conductive cementitious composites can be widely used in the fields of intelligent buildings, health monitoring, disaster and damage prevention, etc. Short carbon fiber [1] was firstly introduced into cementitious composites to function as a smart sensing device and allow non-destructive electrical probing of flaws. Recent researches on the conductive cementitious composites mainly focus on adding new types of conductive fillers [2-5] (e.g. nickel powder [6] and carbon nanotube/nanofiber [7-17]) into the cementitious materials to further modify the sensitivity and stability of the piezoresistive effect. They found that carbon nanotube/nanofiber filled cementitious composites presented obvious piezoresistive effect.

Nano graphite platelets (NGPs) are stack of monolayer carbon atom flat-structure graphene $[18,19]$. They are bidimensional carbon nanomaterials with nano scale thickness and several micrometers diameter, and have one more dimension than carbon nanotube/nanofiber. The extreme case of NGPs is single layer graphene. The electronic mobility of NGPs in ambient temperature exceeds $15000 \mathrm{~cm}^{2} /(\mathrm{V} \cdot \mathrm{s})$ which 
is higher than that of carbon nanotube and silica crystals. As a result, the electrical resistivity of NGPs is only about $10^{-6} \Omega \cdot \mathrm{cm}$, which is lower than that of copper and silver. The connections among carbon atoms in each single layer of NGPs are very pliable and tough. When NGPs are under exterior mechanical force, the carbon atoms will bend and deform without realigning to adapt to the force. This makes their structure remain stable. This steady lattice structure endows carbon atoms with excellent electrical conductivity. Meanwhile, there are no lattice imperfection and exterior atoms introduced in the NGPs. When the electrons are moving in the orbit, they will not scatter. Due to the strong interatomic force, the interference between the electrons in graphene is still very small even if the surrounding carbon atoms collide. These characteristics make NGPs excellent conductive fillers. Previous studies have demonstrated that NGPs exhibit good electrical conductivity and the potential to be used in cementitous composites to monitor the loading, deformation and damage [20], in addition to its benefits on strength, durability and functional properties of the cementitious composites [21]. For example, Le et al. investigated the use of NGPs in cementitious composites to quantify the material damage extent [22]. Huang et al. observed that the addition of NGPs increases the flexural strength of cementitious composites by $82 \%$ compared to the plain ones. The electrical property of the composites is significantly modified, and the composites become highly conductive [23]. Duan et al. reported that only $0.05 \%$ NGPs oxide can improve the flexural strength of cementitious composites from between $41 \%$ to $59 \%$ and the compressive strength from between $15 \%$ to $33 \%$ [24]. Lv et al. found that NGPs oxide nanosheets 
can remarkably increase the tensile/flexural strength of the corresponding cementitious composites. Especially, when the content of NGPs oxide is $0.03 \%$, the tensile, flexural and compressive strength of cementitious composites are increased by $78.6 \%, 60.7 \%$ and $38.9 \%$, respectively [25]. Singh et al. found that the total shielding effectiveness of the NGPs oxide-ferrofluid-cementitious composites is $46 \mathrm{~dB}$, which is much higher than that of the pristine cementitious composites (4dB) [26]. Peyvandi et al. investigated the contributions of NGPs to the durability of cementitious composites, and observed that NGPs significantly improve the moisture transport performance and acid resistance of composites at a low concentration $(0.05$ vol. \% of cementitious composites) [27].

The high electrical conductivity and surface area of NGPs are expected to impart sensitive piezoresistive characteristics to cementitious composites. In this paper, experiments are carried out for verifying this assumption. The cementitious composites filled with NGPs exhibited significant change in resistance when subjected to compressive loadings. The electrical property test and quantitative evaluation of the piezoresistive effect-based sensing capability of cementitious composites filled with different doping levels of NGPs (varying from 0 vol. \% to 10 vol. \% ) under variable loading conditions were performed. Furthermore, the electrically conductive and piezoresistive mechanisms for cementitious composites filled with NGPs under compressive loadings are analyzed through microstructure observation.

\section{Materials and experimental method}




\subsection{Materials}

Cementitious composite are fabricated with cement (type P.O. 42.5R, Dalian Onoda Cement Company), NGPs (shown in Figure 1, and their properties are presented in Table 1., provided by Shanghai Ranen new Energy Equipment \& Technology Co., Ltd.), polycarboxylate superplasticizer (Sika ViscoCrete 3301E, employed to disperse NGPs), and water. The electrical connections are made using $20 \mathrm{~mm} \times 30 \mathrm{~mm}$ stainless steel nets with $2 \mathrm{~mm} \times 2 \mathrm{~mm}$ of mesh size.

Table 1 Properties of NGPs

\begin{tabular}{cccc}
\hline Parameters & Diameter & Thickness & Specific surface area \\
\hline Description/Value & $<2 \mu \mathrm{m}$ & $1-5 \mathrm{~nm}$ & $500 \mathrm{~m}^{2} / \mathrm{g}$ \\
\hline
\end{tabular}

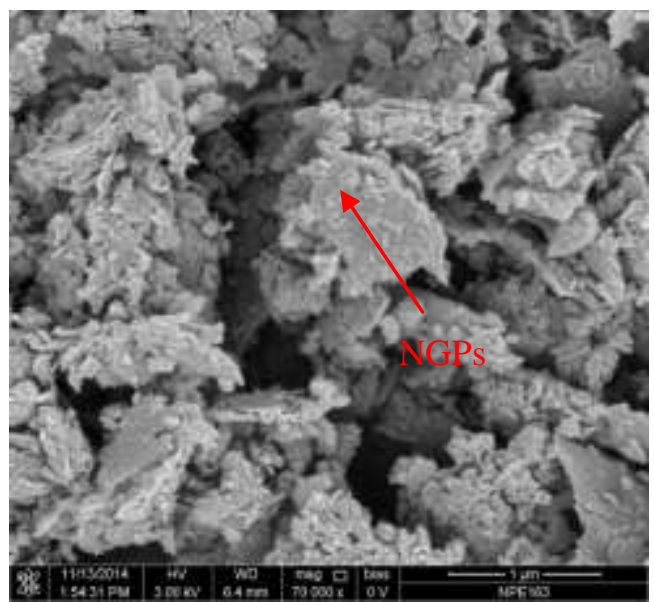

Figure 1 Field emission scanning electron microscope (FESEM) images of NGPs

\subsection{Preparation of cementitious composites filled with NGPs}

The mix proportions of the cementitious composites filled with NGPs are shown in Table 2. When the content of NGPs is more than 6 vol. \%, the workability of the mixture of cementitious composites with water to cement ratio of 0.3 is too poor to mould. Therefore, the ratio of water to cement is raised to 0.4 for cementitious composites with high content of NGPs. The detailed process of 
cementitious composites filled with NGPs is presented in Figure 2: (1) Mix the polycarboxylate superplasticizer with water; (2) Add NGPs into the solution and then ultrasonicate the suspension for 1 hour with an ultrasonicator (American Branson 2510 E-DTH, Bransonic Ultrasonics Corporation) to prepare uniform suspension; (3) Put the cement in batches into the suspension slowly and stir them with a DW-2 DC Constant Speed Stirrer (Yu Hua Instrument Ltd., China) at low speed firstly and then at high speed; (4) Pour the mixture into the mould (20 $\mathrm{mm} \times 20 \mathrm{~mm} \times 40 \mathrm{~mm}$ ) and embed in two electrodes with $10 \mathrm{~mm}$ interval; (5) Put the mould with the mixture on the electric vibrator and vibrate to eliminate bubbles; (6) Demould after 24 hours; (7) Cure the specimens for 28 days in standard condition at a $20^{\circ} \mathrm{C}$ of temperature and a $100 \%$ of relative humidity; (8) Dry the specimens for 30 days at room temperature before testing [28, 29] .

Table 2 Mix proportions of cementitious composites filled with NGPs

\begin{tabular}{cccc}
\hline Sample & NGP content (vol. \%) & Water to cement ratio & Superplasticizer content (vol. \%) \\
\hline NGP-1 & 1 & 0.3 & 1 \\
NGP-2 & 2 & 0.3 & 1 \\
NGP-3 & 3 & 0.3 & 1 \\
NGP-4 & 4 & 0.3 & 1 \\
NGP-5 & 5 & 0.3 & 1 \\
NGP-6 & 6 & 0.4 & 1 \\
NGP-8 & 8 & 0.4 & 1 \\
NGP-9 & 9 & 0.4 & 1 \\
NGP-10 & 10 & 0.4 & 1 \\
\hline
\end{tabular}






Figure 2 Preparation process of NGPs filled cementitious comospites

\subsection{Experimental tests of electrical property and piezoresistive effect of NGPs}

\section{filled cementitious composites}

The electrical resistance without loading is tested by direct current (DC) twoelectrode method using a Digital Multimeter (Keithley 2100 supplied by Keithley Instruments Inc.), as shown in Figure 3.

Compressive loading test is performed by using an Instron 5576A Universal Material Testing Machine. The tested compressive strength of the cementitious composites filled with NGPs is about $80 \mathrm{MPa}$. In order to ensure that the loading amplitude is in elastic regime, $1 / 4$ of the compressive strength i.e. $20 \mathrm{MPa}$ is adopted as the maximum stress for piezoresistive test. The data of the compressive stress was collected by the computer as displayed in Figure 3. During the process of loading, the electrical resistance of the cementitious composites filled with NGPs in compressive direction vertical to the electrodes was tested. Two strain gauges were fixed to the 
opposite sides of the specimen to test the vertical compressive strains. Dynamic strain indicator (DC-204R provided by Tokyo Sokki Kenkyujo Co., Ltd.) is employed to measure the strain value, as presented in Figure 3. The average strain value calculated with two measured strain values is used to describe the piezoresistive relationship between the change in electrical resistivity and strain. The acquisition frequencies of the electrical resistance and compressive strain and stress are the same.

Under cyclic compressive loading with amplitude of $20 \mathrm{MPa}$, quasi static loading rates of $0.1,0.2,0.3$ and $0.4 \mathrm{~mm} / \mathrm{min}$ were used to investigate the effect of loading rate on piezoresistivity of these composites. Under cyclic compressive loading with constant loading rate of $0.2 \mathrm{~mm} / \mathrm{min}$, stress amplitudes of 2.5, 5, 10 and $20 \mathrm{MPa}$ were set to investigate the effect of stress amplitude on piezoresistivity of these composites. Constant loading with amplitude of $20 \mathrm{MPa}$ was performed to study the stability of piezoresistivity of these composites. 10 times of cyclic loadings with stress amplitude of $20 \mathrm{MPa}$ and loading rate of $0.2 \mathrm{~mm} / \mathrm{min}$ were implemented to explore the repeatability of piezoresistivity of these composites. Cyclic dynamic compressive loadings with stress amplitude of $20 \mathrm{MPa}$ were performed to investigate the dynamic piezoresistive response of these composites with the loading rates of $0.8-40 \mathrm{~mm} / \mathrm{min}$ (i.e. loading frequency of $0.1-5 \mathrm{~Hz}$ ), covering the typical frequency content of the dynamic response of large civil engineering structures. Three specimens of each type of cementitious composites were tested for average value. All the experiments were carried out at room temperature. 




Figure 3 Sketch of the measurement system.

Field Emission Scanning Electron Microscope (FESEM) (Nova NanoSEM 450, American FEI Ltd.) is used to observe the morphology of the NGPs and cementitious composites filled with NGPs.

\section{Results and Discussion}

\subsection{Dispersion of NGPs in cementitious matrix}

Figure 4 shows the FESEM images of NGPs filled cementitious composites. The images demonstrate that NGPs are uniformly incorporated into the cementitious matrix. The low errors of the average value of the electrical resistivity in Figure 5 also indicate that the NGPs are uniformly dispersed in the cementitious matrix. 


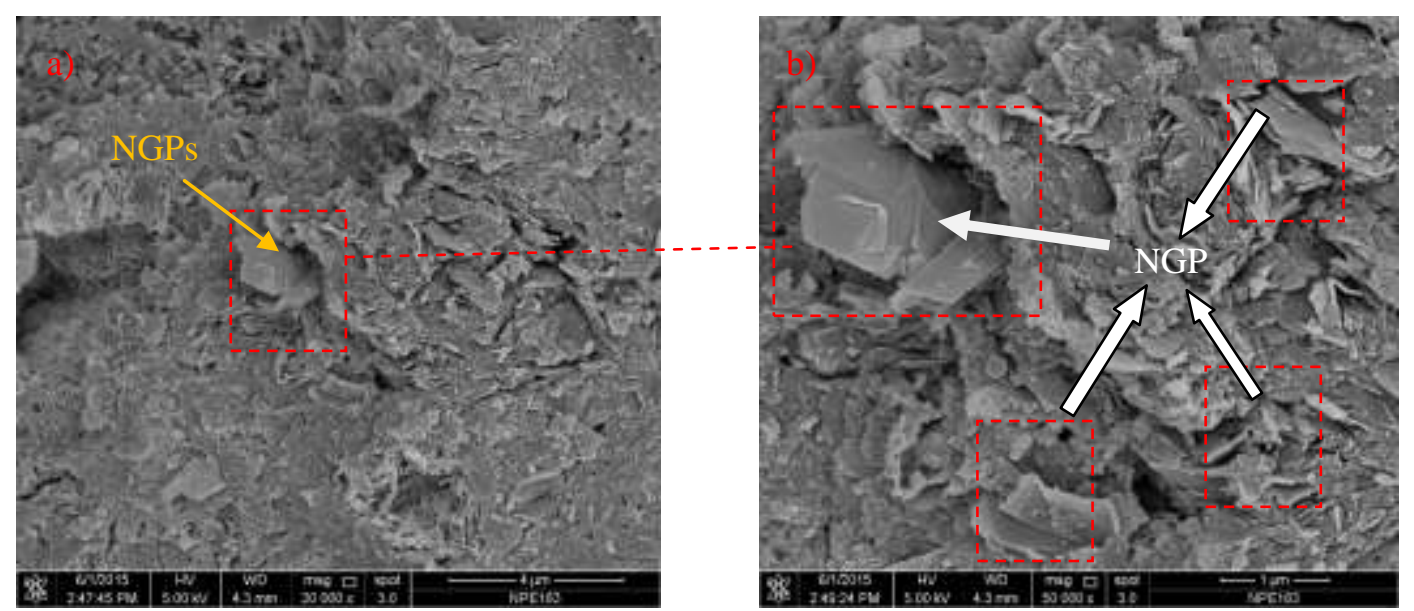

Figure 4 FESEM images: NGPs filled cementitious composites: a) 20000x; b) 50000x

\subsection{Electrical resistance characteristics of cementitious composites filled with}

\section{NGPs}

Figure 5 shows the variation of resistivity of cementitious composites filled with different content levels of NGPs along with test time. It can be seen from Figure 5 that the electrical resistivity increases rapidly at the beginning of the test and then grows slowly. This indicates that there exists polarization effect in these cementitious composites under DC voltage. This phenomenon is similar to the charge of the capacitor, which produces an opposite charging current inside the composites during the electrical resistance test. This leads to the decrease of the current and the increase of the measured electrical resistivity along with test time [30]. The change of the electrical resistivity at the initial stage is too large, which will affect the test of the piezoresistive effect of NGPs filled cementitious composites. As presented in Figure 5 a)-j), the electrical resistivity of the cementitious composites filled with low content of NGPs is hard to be stable. Therefore, as shown in Figure 6 a)-c), the piezoresistive effect of cementitious composites filled with low content of NGPs was tested when the electrical resistance slowly increases along with test time. As shown in Figure 6 
d)-j), the piezoresistive effect of cementitious composites filled with high content of NGPs was tested until the electrical resistance becomes stable along with test time. It can also be seen from Figure 5 that the electrical resistivity of cementitious composites filled with NGPs decreases with the increase of the dosage of NGPs. When the content of NGPs reaches 2 vol. \% the electrical resistivity of the composites decreases to $1 / 4$ of that of the cementitious materials without NGPs. It reveals that the percolation threshold of electrical resistivity of cementitious composites filled with NGPs is nearly 2 vol. \%. When the content of NGPs reaches 9 vol. $\%$ the electrical resistivity of NGPs filled cementitious composites decreases to $1 / 40$ of that of the cementitious materials without NGPs. This indicates that there appears secondary percolation phenomenon as the content of NGPs reaches 8 vol. \%. When the addition of NGPs reaches $10 \mathrm{vol} \%$, the electrical resistivity of NGPs filled cementitious composites is $20 \mathrm{~K} \Omega \cdot \mathrm{cm}$, which decreases to $1 / 120$ of $2400 \mathrm{~K} \Omega \cdot \mathrm{cm}$ of the cementitious materials without NGPs. This makes the conductivity of the cementitious composites suitable for piezoresistive test.
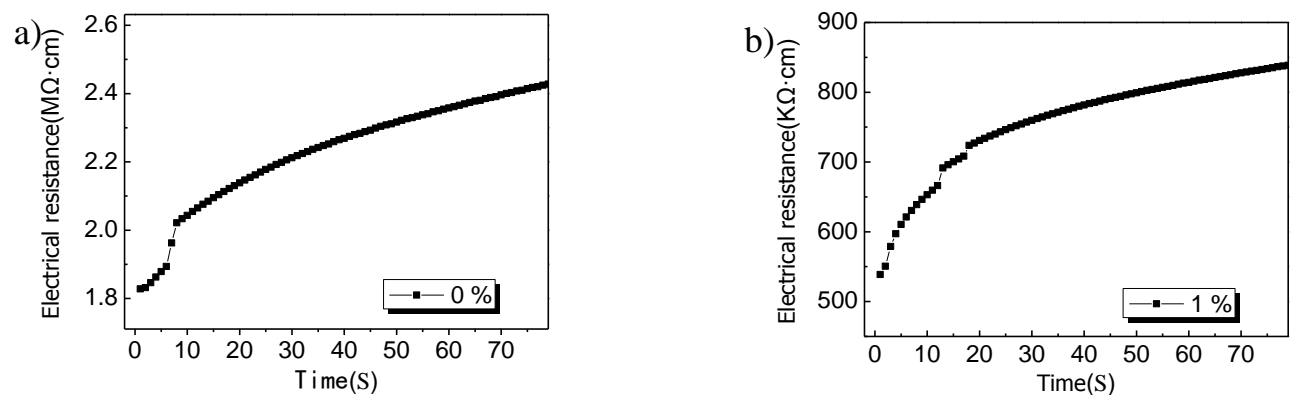


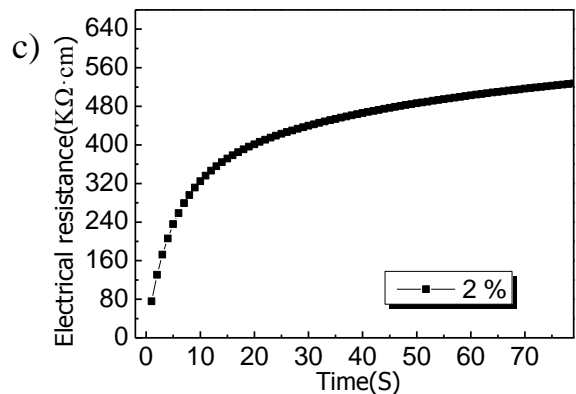

d)

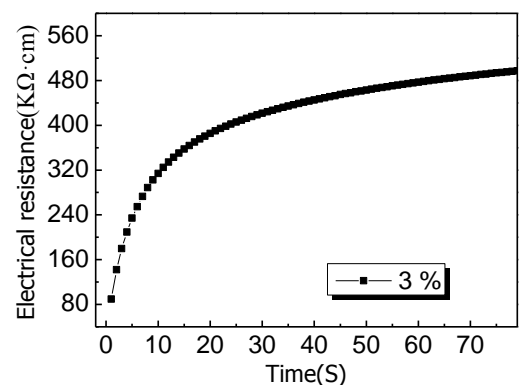

e)



f)

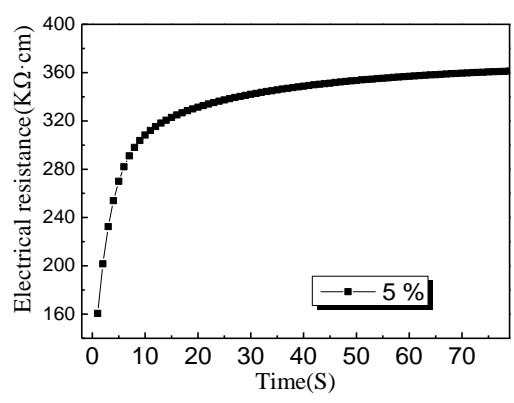

g)
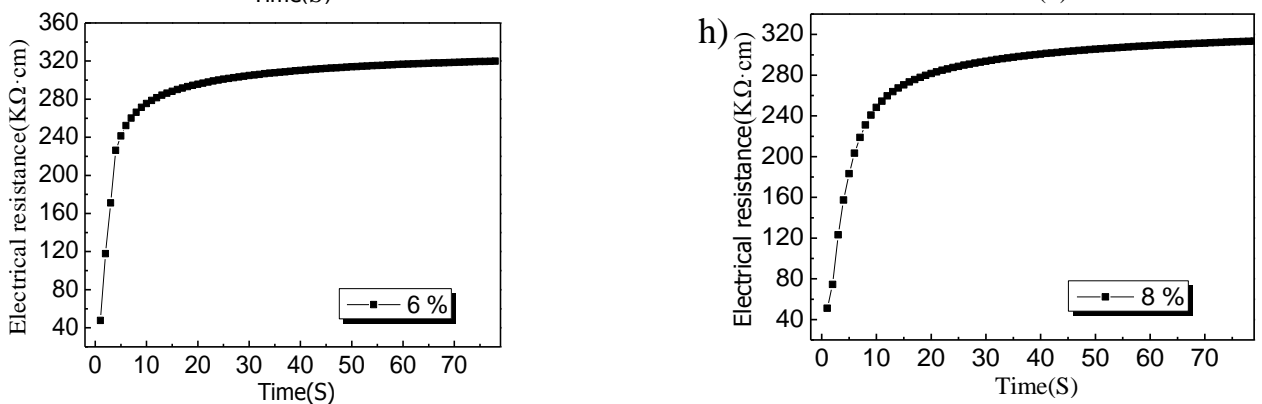

i)
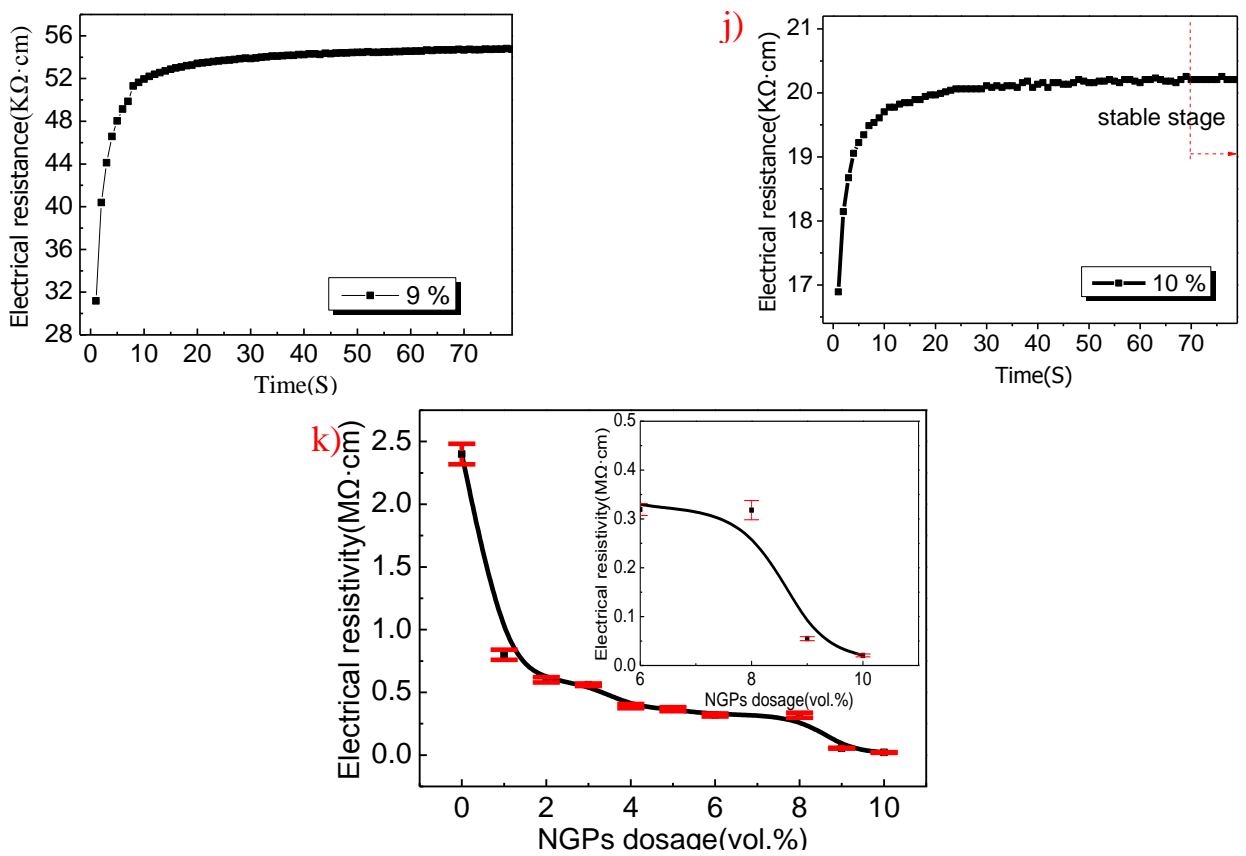

Figure 5 Electrical resistivity of cementitious composites filled with NGPs: a)-j) Electrical resistivity of cementitious composites filled with NGPs 0 vol. \% to 10 vol. \%; k) Relationship between electrical resistivity and NGP content

\subsection{Piezoresistive characteristic of cementitious composites filled with different}




\section{doping levels of NGPs}

Figure 6 shows the fractional change in electrical resistivity of cementitious composites filled with 0 vol. $\%$ to 10 vol. $\%$ of NGPs under cyclic compressive loading. As presented in Figure 6 a) and b), there is no change in electrical resistivity for the composites with low NGP doping levels $(<1 \%)$. This demonstrates that cementitious materials without NGPs and cementitious composites filled with 1 vol. \% of NGPs possess no piezoresistive effect.

Figure $6 \mathrm{c}$ ) and d) present $0.53 \%$ and $2.5 \%$ of change in electrical resistivity cementitious composites filled with 2 vol. $\%$ and 3 vol. $\%$ of NGPs respectively, as the compressive stress reaches $20 \mathrm{MPa}$. This indicates that these two types of composites appear unobvious piezoresistive effect.

Figure 6 e) to j) display $6.7 \%-15.6 \%$ of changes in electrical resistivity when the compressive stress reaches $20 \mathrm{MPa}$, which reveals that cementitious composites filled with 4 vol.\%-10 vol. \% of NGPs present sensitive piezoresistive effect. In addition, it can be seen from Figure 6 that the piezoresistive effect of cementitious composites filled with 0 vol. \%-10 vol. \% of NGPs appears a trend of increase at first and then slow decrease. The piezoresistive response of cementitious composites filled with 5 vol. \% of NGPs is the most sensitive among all composites. The piezoresistivities of cementitious composites filled with 9 vol. $\%$ and 10 vol. $\%$ of NGPs are better than that of cementitious composites filled with 2 vol. $\%$ to 4 vol.\% of NGPs, but worse than that of cementitious composites filled with 6 vol. $\%$ and 8 vol. $\%$ of NGPs. 

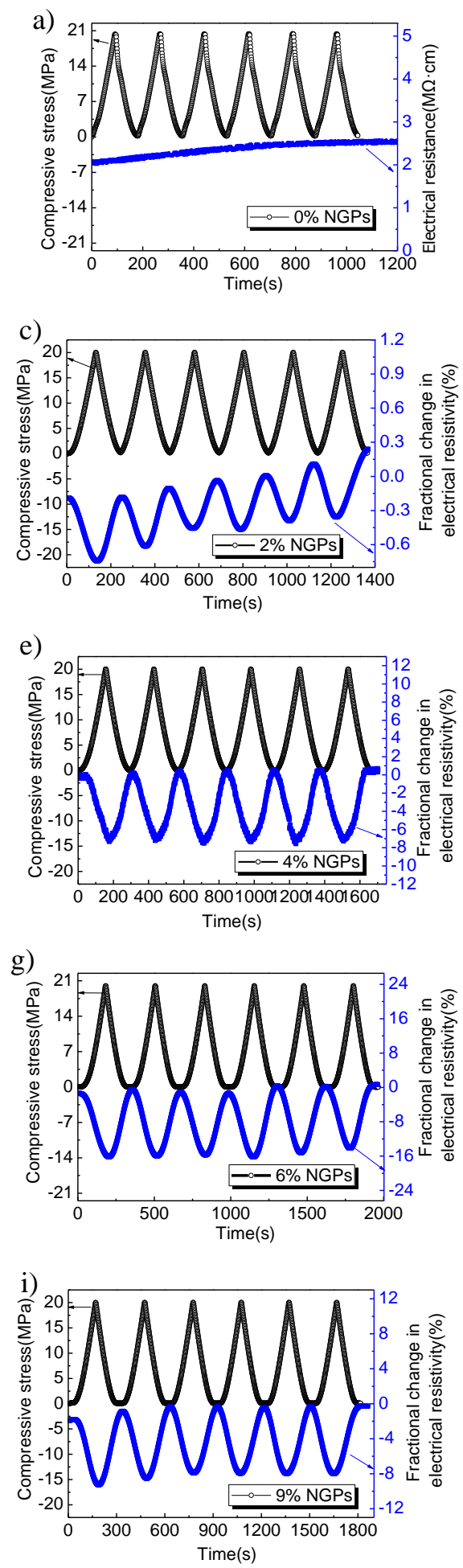
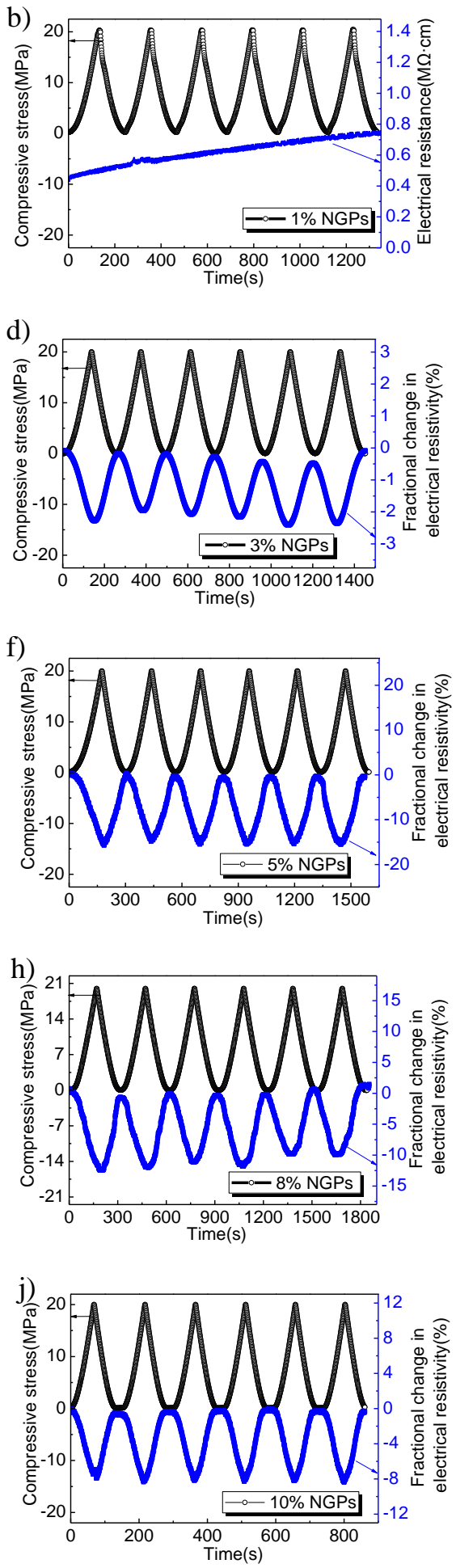


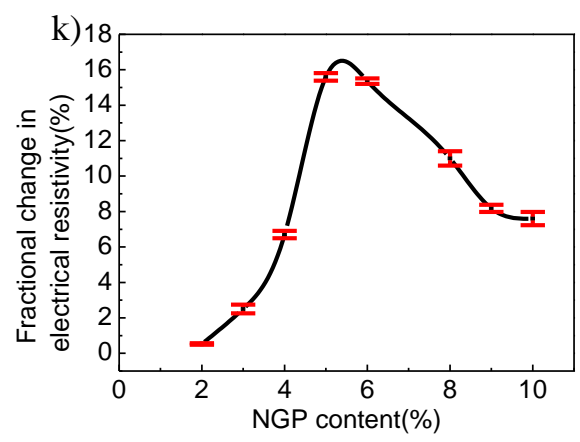

Figure 6 a)-j) Fractional change in electrical resistivity of cementitious composites filled with NGPs of 0 vol.\% to 10 vol.\%; k) Relationship between the highest fractional change in electrical resistivity and NGP content

Figure 7 presents the relationship between fractional change in electrical resistivity and compressive stress under cyclic loading with polynomial fitting. The fractional change in electrical resistivity of the composites is well correlated to the cyclic loading except for that of cementitious composites filled with 2 vol. $\%$ of NGPs. The cementitious composites with 5 vol. $\%$ of NGPs have the highest sensitivity to compressive stress, which is $0.78 \% / \mathrm{MPa}$. This sensitivity is higher than that of the cementitious composites filled with carbon nanotube/nanofiber, which is $0.225 \% / \mathrm{MPa}$ [31].
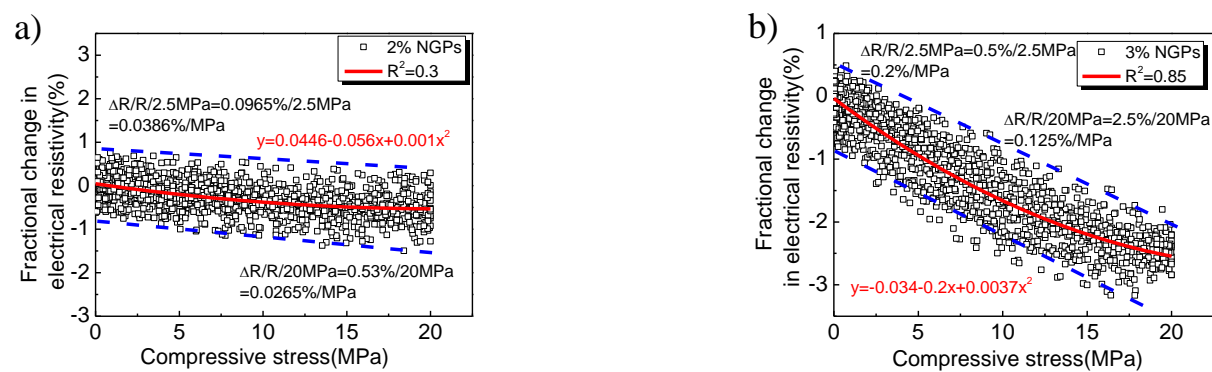

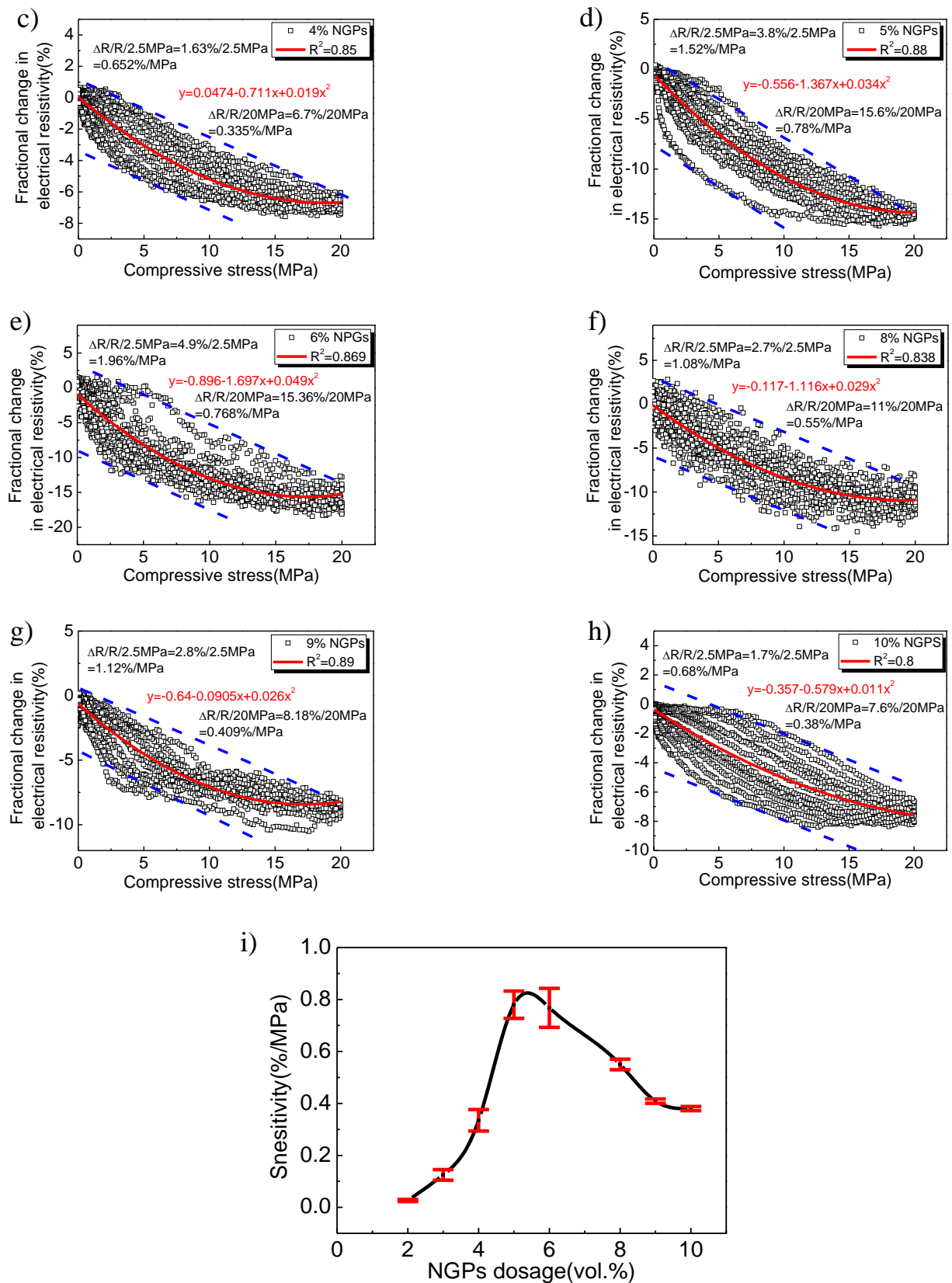

Figure 7 a)-h) Fractional change in electrical resistivity of cementitious composites filled with NGPs of 0 vol.\% to 10 vol.\%; i) Relationship of sensitivity and NGP content

The response of the fractional change in electrical resistivity to compressive strain is almost the same as the results presented in Figure 6. Because the relationship between compressive stress and strain of the cementitous composites in the elastic range is linear, the response of the fractional change in electrical resistivity to the 
compressive strain is consistent with the response of the fractional change in electrical resistivity to the compressive stress. The strain sensitivity (i.e. gauge factor) of cementitious composites filled with 0 vol. \%-10 vol. \% of NGPs appears a trend of increase at first and then slow decrease too. The highest gauge factor of the cementitious composites filled with 5 vol. \% of NGPs is 156 , which is far more than 2-3 of metal foil strain gauge. The gauge factors obtained from this study are compared with that reported in the literature as presented in Table 3 [32]. Moreover, the repeatability and stability of the cementitious composites filled with NGPs are better than that in the literature. It can be seen from Table 3 that the developed composites with NGPs in this paper have much higher gauge factors than the composites with NGPs in the reference [19].

Table 3 Comparison of gauge factors

\begin{tabular}{cccc}
\hline Matrix & Filler & Gauge factor & Resource \\
\hline Fly ash geopolymeric composites & NGPs oxide & 36.9 & Reference 19 \\
NGPs filled cementitious composites & NGPs & 156 & In this paper \\
\hline
\end{tabular}

\subsection{Piezoresistive effect of cementitious composites filled with NGPs under different loading conditions}

Because the piezoresistive effect of cementitious composites with 5 vol. \% of NGPs is the best among all composites, these cementitious composites are employed to test the piezoresistive effect under different loading conditions.

Figure 8 shows the fractional change in electrical resistivity along with time under cyclic variable loading rates with an amplitude of $20 \mathrm{MPa}$ loading stress. As presented 
in Figure 8 a), the amplitudes of fractional change in electrical resistivity do not change at different loading rates.

Figure $8 \mathrm{~b}$ ) displays the response of the fractional change in electrical resistivity to the compressive strain under cyclic variable loading rates. The response of fractional change in electrical resistivity to the compressive strain is consistent with the fractional change in electrical resistivity to the compressive stress presented in Figure 8 a).
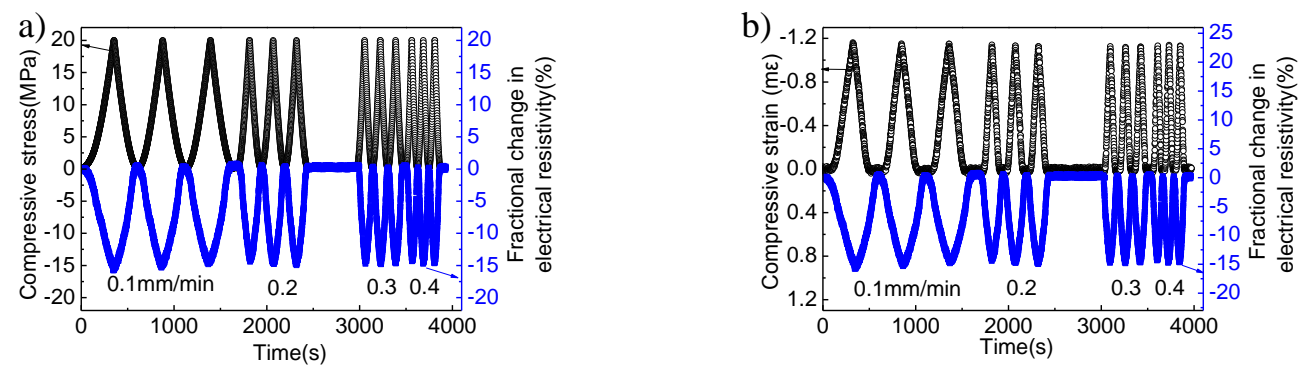

Figure 8 a) Fractional change in electrical resistivity along with time under cyclic variable loading rate; b) Response of fractional change in electrical resistivity to compressive strain along with time under cyclic variable loading rate

The response of the fractional change in electrical resistivity to compressive stress and strain along with time under cyclic variable amplitudes loading with a constant loading rate of $0.2 \mathrm{~mm} / \mathrm{min}$ is presented in Figure 9. The amplitude of fractional change in electrical resistivity increases as compressive stress and strain amplitudes increase. The fractional change in electrical resistivity can reach $2.9 \%, 6.2 \%, 10.4 \%$ and $15.6 \%$ when the compressive stress is $2.5,4,10$ and $20 \mathrm{MPa}$, respectively. The fractional change in electrical resistivity of the composites shows stable and sensitive response to different loading amplitudes. 

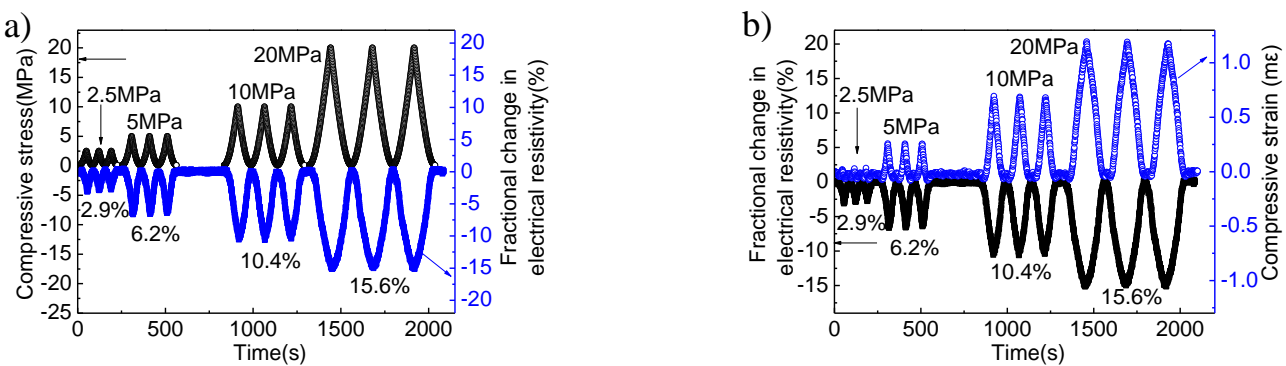

Figure 9 a) Fractional change in electrical resistivity along with time under cyclic variable amplitude loading; b) Response of fractional change in electrical resistivity to compressive strain along with time under cyclic variable amplitude loading

Figure 10 displays the response of the fractional change in electrical resistivity to compressive stress and strain along with time under cyclic constant loading with an amplitude of $20 \mathrm{MPa}$ loading stress, respectively. The fractional change in electrical resistivity keeps unchanged when the compressive stress and strain remain constant. It is revealed that the piezoresistive effect of the cementitious composites filled with NGPs is stable.
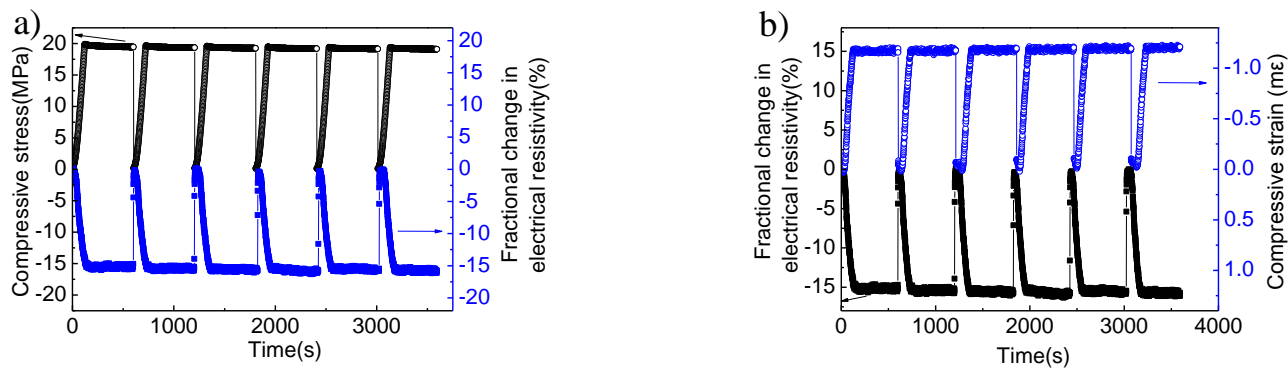

Figure 10 a) Fractional change in electrical resistivity along with time under cyclic constant loading; b) Response of fractional change in electrical resistivity to compressive strain along with time under cyclic constant loading

The fractional change in electrical resistivity to the compressive stress and the compressive strain along with time under 10 times of cyclic loading with an amplitude of $20 \mathrm{MPa}$ loading stress at a constant loading rate of $0.2 \mathrm{~mm} / \mathrm{min}$ after 40 
times cyclic compressive loading is presented Figure 11. As shown in Figure 11, the piezoresistive effect of cementitious composites filled with NGPs presents stable repeatability after 40 times of repeated cyclic $20 \mathrm{MPa}$ loading.
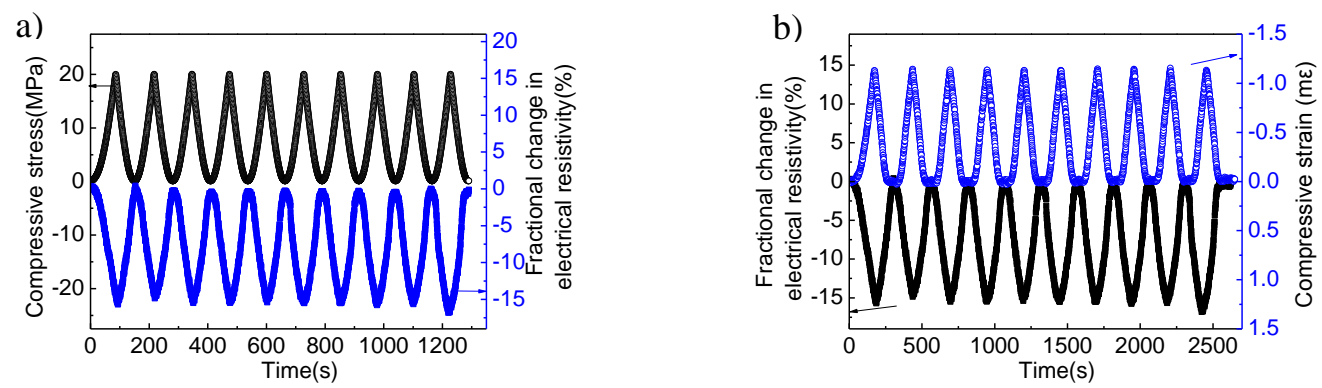

Figure 11 a) Fractional change in electrical resistivity along with time under 10 times of cyclic loading; b) Response of fractional change in electrical resistivity to compressive strain along with time under 10 times of cyclic loading after 40 times of cyclic loading

\subsection{Piezoresistive effect of cementitious composites filled with NGPs under}

\section{dynamic loading conditions}

Figure 12 shows the fractional change in electrical resistivity with respect to different dynamic loading rates. The fractional change in electrical resistivity performs stable consistency to the dynamic cyclic loading. And the response of the fractional change in electrical resistivity to the dynamic cyclic loading presents stable repeatability.
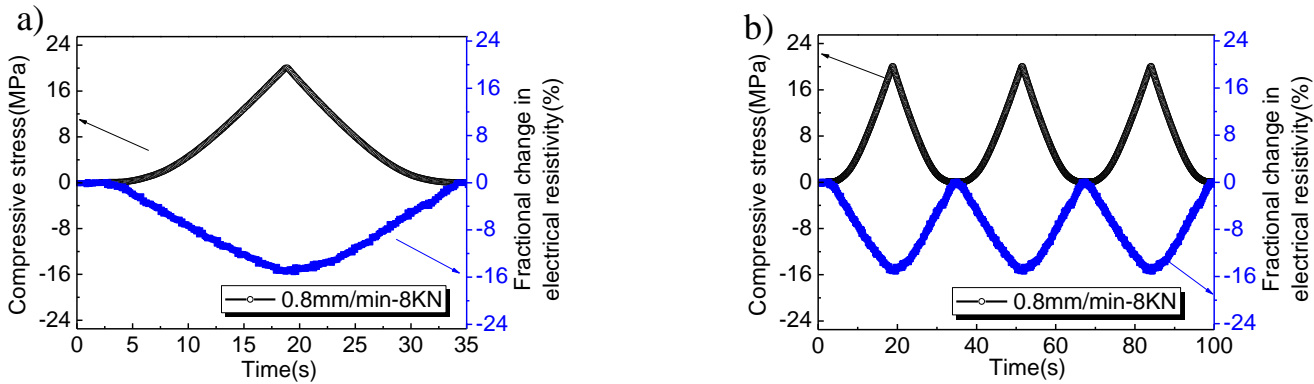

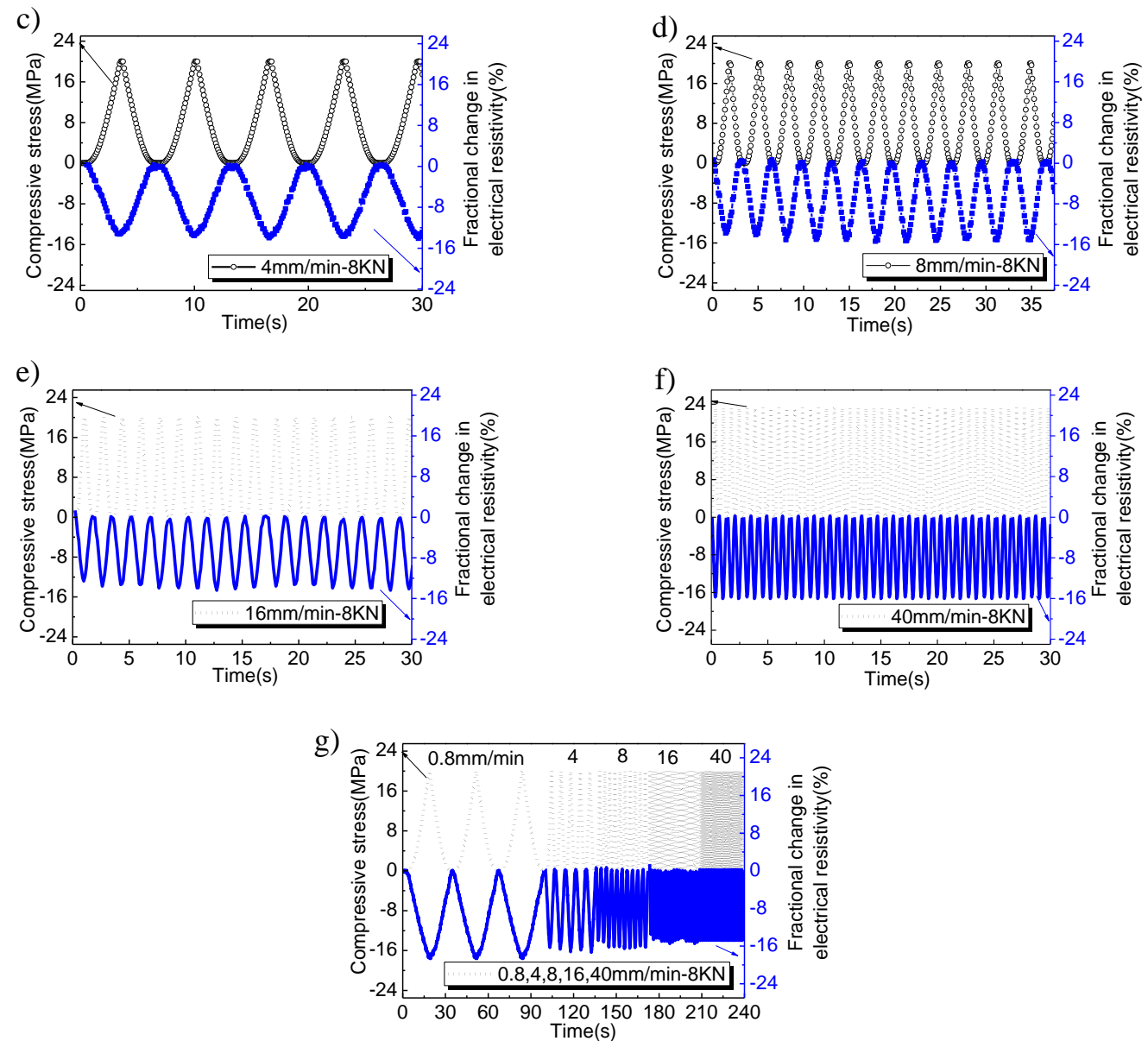

Figure 12 Fractional change in electrical resistivity of cementitious composites filled with NGPs under different dynamic cyclic loading rates: a) $0.8 \mathrm{~mm} / \mathrm{min}(30 \mathrm{~s})$, b) $0.8 \mathrm{~mm} / \mathrm{min}(100 \mathrm{~s})$, c) $4 \mathrm{~mm} / \mathrm{min}(30 \mathrm{~s})$, d) $8 \mathrm{~mm} / \mathrm{min}(30 \mathrm{~s})$, e) $16 \mathrm{~mm} / \mathrm{min}(30 \mathrm{~s})$, f) $40 \mathrm{~mm} / \mathrm{min}(30 \mathrm{~s}), \mathrm{g})$

$0.8,4,8,16,40 \mathrm{~mm} / \mathrm{min}$

Figure 13 gives the response of fractional change in electrical resistivity to compressive strain along with time under different dynamic cyclic loading rates. The fractional change in electrical resistivity performs stable consistency to the compressive strain under different dynamic cyclic loading rates. The response of the fractional change in electrical resistivity to the compressive strain under dynamic cyclic loading presents stable repeatability too. However, the amplitudes of change in the electrical resistivity show a little decrease along with test time. This behavior displays a slight dynamic loading rate dependency of the change amplitudes of the 
electrical resistivity.
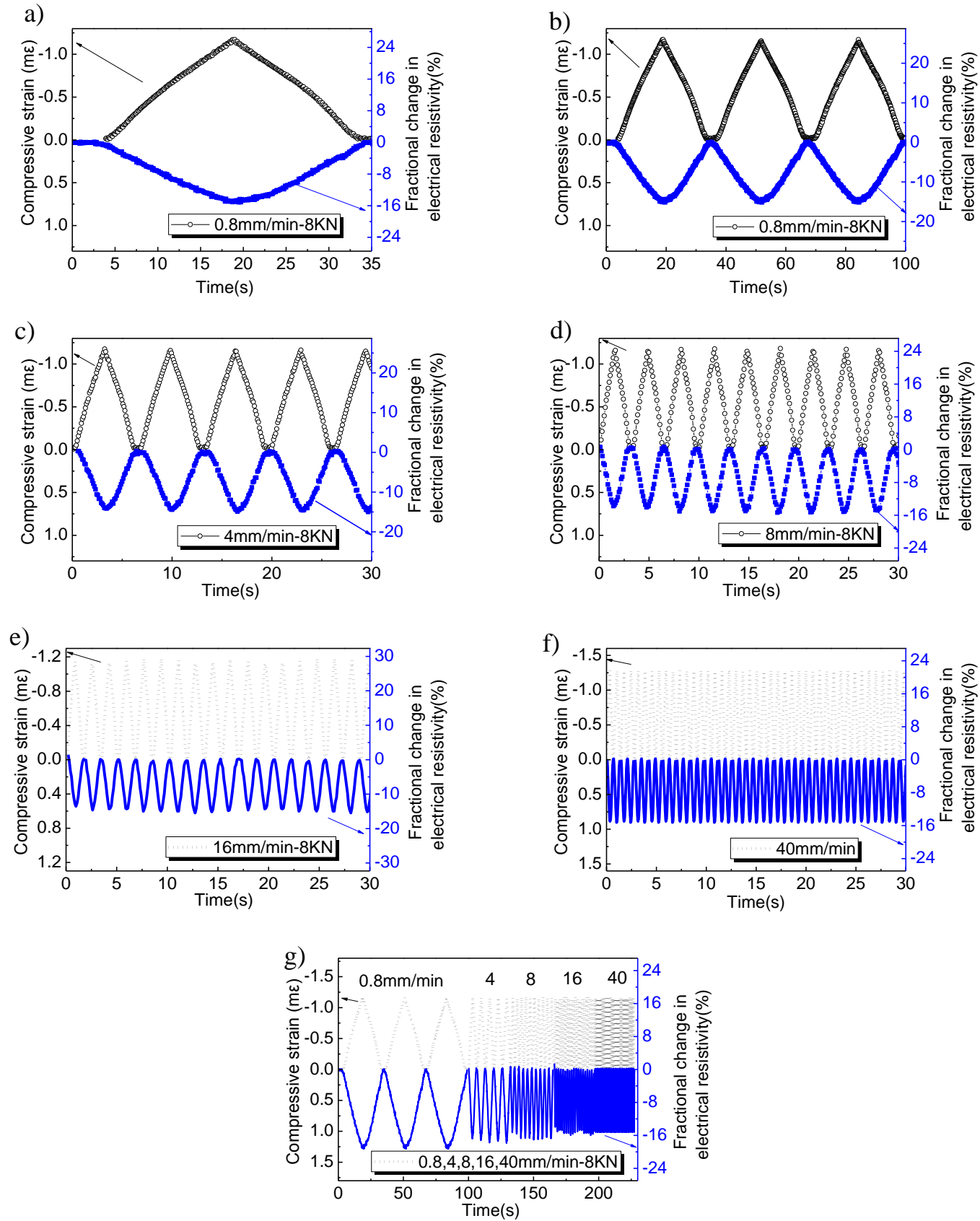

Figure 13 Response of fractional change in electrical resistivity to compressive strain of cementitious composites filled with NGPs along with time under dynamic cyclic loading: a) $0.8 \mathrm{~mm} / \mathrm{min}(30 \mathrm{~s})$, b) $0.8 \mathrm{~mm} / \mathrm{min}(100 \mathrm{~s})$, c) $4 \mathrm{~mm} / \mathrm{min}(30 \mathrm{~s})$, d) $8 \mathrm{~mm} / \mathrm{min}(30 \mathrm{~s})$, e) $16 \mathrm{~mm} / \mathrm{min}$ (30s), f) $40 \mathrm{~mm} / \mathrm{min}(30 \mathrm{~s})$, g) $0.8,4,8,16,40 \mathrm{~mm} / \mathrm{min}$

4 Mechanisms of electrical conductivity and piezoresistivity of cementitious composites filled with NGPs 


\subsection{Mechanisms of electrical conductivity}

NGPs have pi-electrons that participate in interlayer pi bonding. This makes NGPs good electrical conducting characteristic. Besides, the carbonation process with temperature up to $1050^{\circ} \mathrm{C}$ during fabrication of NGPs produces a large excess of holes in the valence band. These characteristics of NGPs contribute to the electrical conductivity and piezoresistivity of cementitious composites filled with NGPs through the following paths: (1) Electronic conduction through NGPs by tunneling effect; (2) Electronic conduction and hole conduction through contacting conduction of NGPs (as shown in Figure 15). When the content of NGPs is within percolation zones, NGPs filled in the cementitious composites form conductive networks through the above two paths, which leads to the obvious decrease of electrical resistivity as shown in Figure 14 c) to j). However, when the content of NGPs is lower than percolation threshold, the separation between NGPs is too far. The NGPs in the cementitious composites cannot form conductive networks. As shown in Figure 14 a) and b), the electrical resistivity of cementitious composites filled with 0 vol. $\%$ and 1 vol. $\%$ of NGPs is much higher than that of cementitious composites filled with NGPs whose content levels are within percolation zones. 

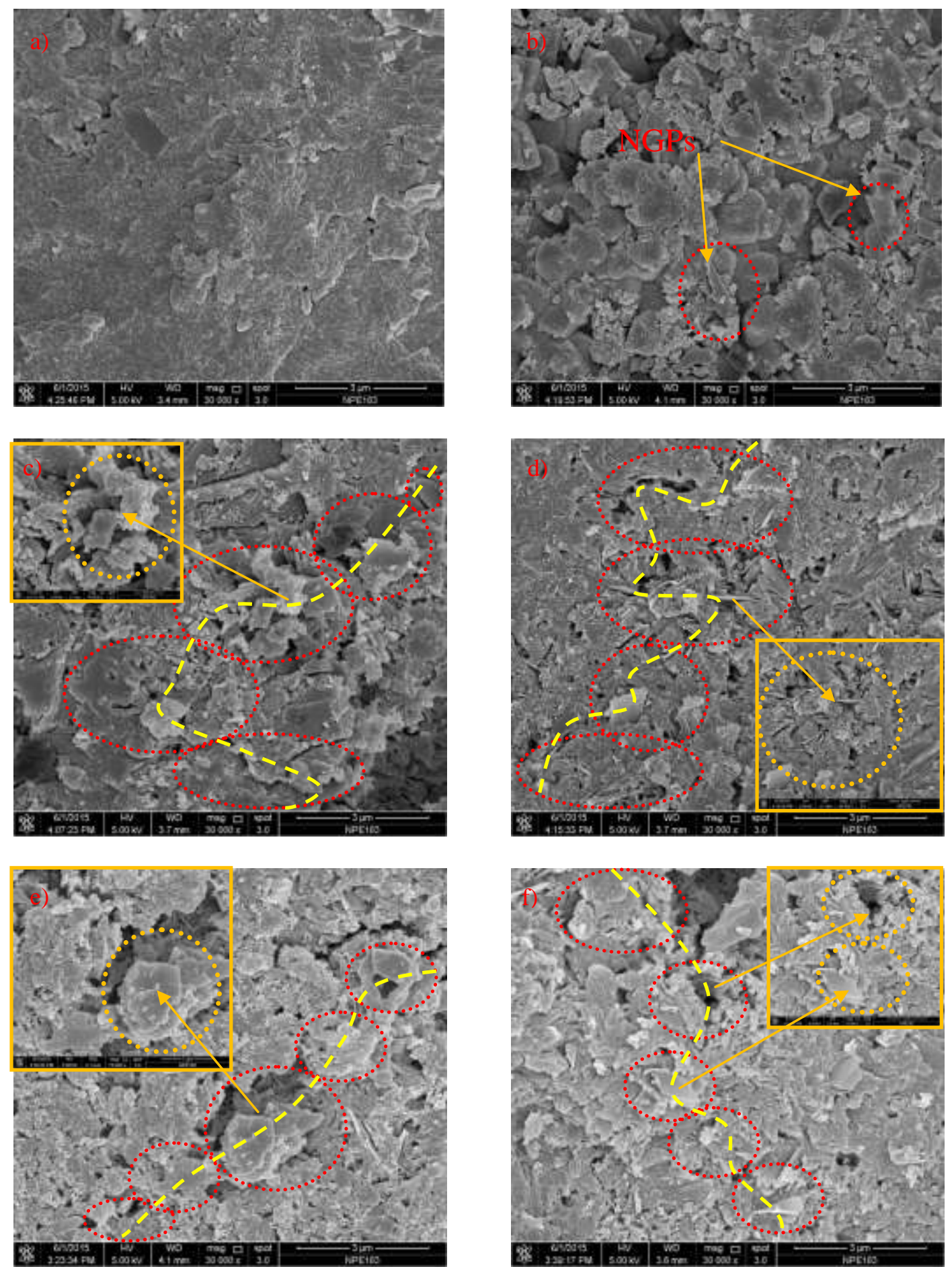

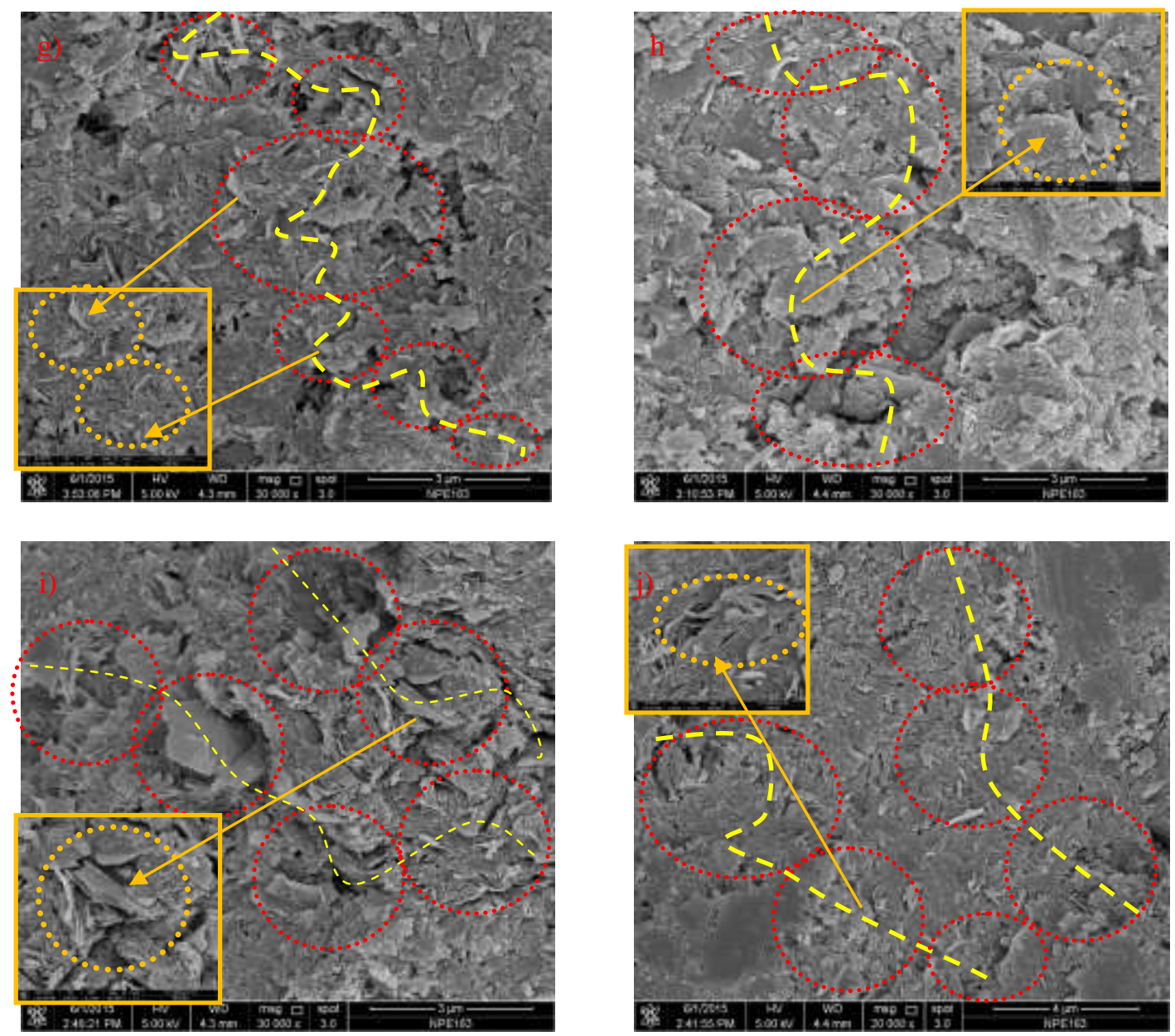

Figure 14 Cementitious composites filled with a) 0 vol. \% NGPs; b) 1 vol. \% NGPs; c) 2 vol. \% NGPs: 30000x and 100000x (on the top left); d) 3 vol. \% NGPs : 30000x and 50000x (on the bottom right); e) 4 vol. \% NGPs: 30000x and 70000x (on the top left); f) 5 vol. \% NGPs: 30000x and 70000x (on the top right); g) 6 vol. \% NGPs: 30000x and 70000x (on the bottom left); h) 8 vol. \% NGPs: 30000x and 100000x (on the top right); i) 9 vol. \% NGPs: 30000x and 100000x (on the bottom left); j) 10 vol. \% NGPs: 20000x and 100000x (on the top left)

\subsection{Mechanisms of piezoresistivity}

When the content of NGPs is lower than percolation threshold, the separation between NGPs in the cementitious composites is too far. The NGPs cannot contact each other when the composites are deformed by compressive loading. Therefore, there is no change in electrical resistivity for the composites with 0 vol. $\%$ and 1 vol. $\%$ of NGPs as they do not fall in the percolation zone. When the content of NGPs reaches percolation threshold, NGPs filled in the cementitious composites form 
conductive networks. The distance between NGPs and their multi layers will be reduced as the cementitious composites filled with NGPs are deformed under compressive loading. As a result, it is easier for electron migration between NGPs and their multi layers as presented in Figure 15, which leads to the enhancement of tunneling conduction. Therefore, the electrical resistivity of the cementitious composites filled with NGPs decreases under external load, thus expressing stable and strong stress/strain sensing responses [33-37].

A higher content of NGPs can make more stable conductive networks formed by the NGPs in the cementitious composites and higher probability of tunneling conduction occurrence under external load. Therefore, the response of the change in electrical resistivity of the cementitious composites filled with 5 vol. \% NGPs to compressive stress/strain is stable under different loading conditions. However, the NGPs show a trend to aggregate and cluster when the content of NGPs exceeds 5 vol.\%. This will lower the piezoresistive effect of the cementitious composites filled with 6 vol. $\%$ to 10 vol.\% NGPs compared to the cementitious composites filled with 5 vol.\% NGPs. Therefore, the cementitious composites filled with 5 vol.\% NGPs present the best piezoresistive effect among all composites.

When the loading rates change from $0.8 \mathrm{~mm} / \mathrm{min}$ to $40 \mathrm{~mm} / \mathrm{min}$ under dynamic loading, the compressive strain of the cementitious composites at compressive stress of $20 \mathrm{MPa}$ presents a little decrease. This means the deformation of the composites can't catch up with the loading at high loading rate. Therefore, the amplitude of fractional change in electrical resistivity shows a small decrease as shown in Figure 


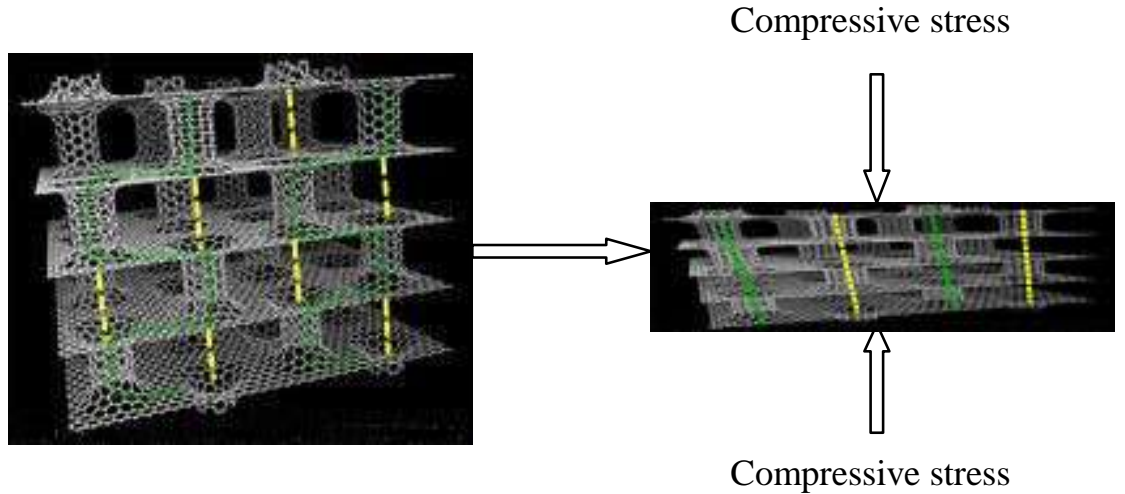

Figure 15 Schematic presentation of the electrical conduction mechanisms of NGPs (yellow and green lines represent conducting pathway)

\section{Conclusions}

In this paper, the electrically conductive and piezoresistive behaviors of cementitious composites filled with 0 vol. $\%$ to 10 vol. \% of NGPs were investigated under variable loading conditions. The percolation threshold of the electrical resistivity of the cementitious composites filled with NGPs is about 2 vol. \% NGPs. There appears second percolation phenomenon as the content of NGPs reaches 9 vol. \%. Cementitious materials without NGPs and cementitious composites filled with 1 vol. \% of NGPs possess no piezoresistive effect. Cementitious composites filled with 2 vol. \% of NGPs showed unobvious piezoresistive effect. The fractional change in electrical resistivity of cementitious composites filled with 4 vol. \%-10 vol. \% of NGPs can reach $6.7 \%$ to $15.6 \%$ when the compressive stress is $20 \mathrm{MPa}$, which reveals that cementitious composites filled with 4 vol. $\%-10$ vol. $\%$ of NGPs show sensitive piezoresistive effect. The cementitious composites filled with 5 vol. \% of NGPs have 
the most sensitive piezoresistivity among all composites. Their fractional changes in electrical resistivity reach $15.6 \%$ when the compressive stress is $20 \mathrm{MPa}$. The highest sensitivities of the cementitious composites filled with NGPs to compressive stress and compressive strain are $0.78 \% / \mathrm{MPa}$ and 156 , respectively. The stress/strain sensitivities of cementitious composites filled with 0 vol. \%-10 vol. \% of NGPs appear a trend of first raise and then slow decrease. The piezoresistive effect of cementitious composites filled with NGPs presents stable repeatability under lots of times of cyclic quasi-static compressive loading and dynamic cyclic loading within elastic regime. However, the amplitudes of change in the electrical resistivity under different dynamic loading rates show a little decrease with test time at high loading rate, which displays a slight dynamic loading rate dependency. The change of electrical resistivity of the cementitious composites filled with NGPs is related to the change of the separation between NGPs and their multi layers under external load. The separation between NGPs and their multi layers will be reduced, i.e. the tunneling conduction is enhanced, when the cementitious composites filled with NGPs are deformed under compressive loading. This leads to stable and strong stress/strain sensing responses of the composites. In addition, the influence of tunneling conduction of cementitious composites filled with NGPs on the piezoresistive effect depends on the content levels of NGPs. When the content of NGPs is within the percolation zones, the cementitious composites filled with NGPs present sensitive piezoresistive effect.

Owing to excellent self-sensing properties, the developed cementitious 
composites filled with NGPs can be considered prototype sensors that appear to be suitable for the health monitoring and state assessment of concrete structures.

\section{Acknowledgments}

The authors thank the funding supported from the National Science Foundation of China (51428801 and 51578110).

\section{References:}

[1] B. Han, X. Yu, J. Ou. Self-sensing concrete in smart structures. Elsevier. 2014: 385.

[2] R. Tawie, H.K. Lee, S.H. Park, R. Tawie, H.K. Lee, S.H. Park. Non-destructive evaluation of concrete quality using PZT transducers. Smart Struct Syst, 2010;6(7):851-66.

[3] R. Tawie, H.K. Lee. Characterization of cement-based materials using a reusable piezoelectric impedance-based sensor. Smart Mater Struct, 2011;20(8):85023-31.

[4] F.N. Meyers, K.J. Loh, J.S. Dodds, B. Arturo. Active sensing and damage detection using piezoelectric zinc oxide-based nanocomposites. Nanotechnology, 2013;24(18):185501.

[5] Y. Zhao, B.R. Loyola, K.J. Loh. Characterizing the viscoelastic properties of layer-by-layer carbon nanotube-polyelectrolyte thin films. Smart Mater Struct, 2011;20(7):75020-30.

[6] B. Han, Y. Yu, B. Han, J. Ou. Development of a wireless stress/strain 
measurement system integrated with pressure-sensitive nickel powder-filled cementbased sensors. Sensor Actuat A-Phys, 2008;147(2):536-43.

[7] F. Azhari, N. Banthia. Cement-based sensors with carbon fibers and carbon nanotubes for piezoresistive sensing. Cement Concrete Comp, 2012;34(7):866-73.

[8] A. Materazzi, F. Ubertini, A. D'Alessandro. Carbon nanotube cement-based transducers for dynamic sensing of strain. Cement Concrete Comp, 2013;37:2-11.

[9] O. Galao, F. Baeza, E. Zornoza, P. Garcés. Strain and damage sensing properties on multifunctional cement composites with CNF admixture. Cement Concrete Comp, 2014;46:90-8.

[10] A. D Alessandro, F. Ubertini, A.L. Materazzi, S. Laflamme, M. Porfiri. Electromechanical modelling of a new class of nanocomposite cement-based sensors for structural health monitoring. Struct Health Monit, 2014:1-11.

[11] F. Ubertini, S. Laflamme, H. Ceylan, A.L. Materazzi, G. Cerni, H. Saleem, et al. Novel nanocomposite technologies for dynamic monitoring of structures: A comparison between cement-based embeddable and soft elastomeric surface sensors. Smart Mater Struct, 2014;23(4):12p.

[12] S. Cardoso, V. Chalivendra, A. Shukla, S. Yang. Damage detection of rubber toughened nanocomposites in the fracture process zone using carbon nanotubes. Eng Fract Mech, 2012;96:380-91.

[13] V. Vadlamani, V. Chalivendra, A. Shukla, S. Yang. In situ sensing of non-linear deformation and damage in epoxy particulate composites. Smart Mater Struct, 2012;21(7):10p. 
[14] N. Heeder, A. Shukla, V. Chalivendra, S. Yang, K. Park. Electrical response of carbon nanotube reinforced nanocomposites under static and dynamic loading. Exp Mech, 2012;52(3):315-22.

[15] N. Heeder, A. Shukla, V. Chalivendra, S. Yang. Sensitivity and dynamic electrical response of CNT-reinforced nanocomposites. J Mater Sci, 2012;47(8):380816.

[16] S. Cardoso, C. O'Connell, R. Pivonka, C. Mooney, V. Chalivendra, A. Shukla, et al. Effect of external loads on damage detection of rubber-toughened nanocomposites using carbon nanotubes sensory network. Polym Composite, 2014:10p.

[17] V. Vadlamani, V. Chalivendra, A. Shukla, S. Yang. Sensing of damage in carbon nanotubes and carbon black-embedded epoxy under tensile loading. Polym Composite, 2012;33(10):1809-15.

[18] S.J. Wang, Y. Geng, Q. Zheng, J.K. Kim. Fabrication of highly conducting and transparent graphene films. Carbon, 2010;48(6):1815-23.

[19] Q. Zheng, Y. Geng, S. Wang, Z. Li, J.K. Kim. Effects of functional groups on the mechanical and wrinkling properties of graphene sheets. Carbon, 2010;48(15):431522.

[20] J. Le, S. Pang, H. Du. Using graphite nanoplatelet reinforced cementitious composites as a self-sensing material: Theory and experiments. EMI 2013 Conference, Aug; 2013; 2013. p. 4-7.

[21] H. Du, S. Pang, S. Quek. Transport properties of cement mortar with graphite nanoplatelet. ICCE-20, Jul, 2012:22-8. 
[22] J. Le, H. Du, S. Dai Pang. Use of 2D Graphene Nanoplatelets (GNP) in cement composites for structural health evaluation. Comos Part B-Eng, 2014;67:555-63.

[23] S. Huang. Multifunctional graphite nanoplatelets (GNP) reinforced cementitious composites. Singapore, National University of Singapore, Master thesis, 2012.

[24] Z. Pan, W. DUAN, D. Li, F. Collins. Graphene oxide reinforced cement and concrete. AU patent 096990. 2013.

[25] S. Lv, Y. Ma, C. Qiu, T. Sun, J. Liu, Q. Zhou. Effect of graphene oxide nanosheets of microstructure and mechanical properties of cement composites. Constr Build Mater, 2013;49:121-7.

[26] A.P. Singh, M. Mishra, A. Chandra, S.K. Dhawan. Graphene oxide/ferrofluid/cement composites for electromagnetic interference shielding application. Nanotechnology, 2011;22(46):9p.

[27] A. Peyvandi, P. Soroushian, A.M. Balachandra, K. Sobolev. Enhancement of the durability characteristics of concrete nanocomposite pipes with modified graphite nanoplatelets. Constr Build Mater, 2013;47:111-7.

[28] B. Han, X. Yu, J. Ou. Effect of water content on the piezoresistivity of MWNT/cement composites. J Mater Sci, 2010;45(14):3714-9.

[29] S. Sun, X. Yu, B. Han. Sensing mechanism of Self-Monitoring CNT cementitious composite. J Test Eval, 2014;42(1):5p.

[30] B. Han, K. Zhang, X. Yu, E. Kwon, J. Ou. Electrical characteristics and pressuresensitive response measurements of carboxyl MWNT/cement composites. Cement Concr Compos, 2012;34(6):794-800. 
[31] A. D'Alessandro, F. Ubertini, S. Laflamme, M. Rallini ; A. L. Materazzi, J. M. Kenny. Strain sensitivity of carbon nanotube cement-based composites for structural health monitoring. Proc. SPIE 9803, Sensors and Smart Structures Technologies for Civil, Mechanical, and Aerospace Systems 2016, 980319 (April 20, 2016). doi:10.1117/12.2218905.

[32] M. Saafi, L. Tang, J. Fung, M. Rahman, F. Sillars, J. Liggat, et al. Graphene/fly ash geopolymeric composites as self-sensing structural materials. Smart Mater Struct, 2014;23(6):10p.

[33] G. Li, P. Wang, X. Zhao. Pressure-sensitive properties and microstructure of carbon nanotube reinforced cement composites. Cement Concr Compos, 2007;29(5):377-82.

[34] B. Han, X. Yu, E. Kwon. A self-sensing carbon nanotube/cement composite for traffic monitoring. Nanotechnology, 2009;20(44):5p.

[35] F. Azhari. Cement-based sensors for structural health monitoring. Canada, University of British Columbia, Master thesis, 2008.

[36] B. Han, S. Sun, S. Ding, L. Zhang, X. Yu, J. Ou. Review of nanocarbonengineered multifunctional cementitious composites. Compos Part A-Appl S, 2015;70:69-81.

[37] M.Q. Sun, R.J. Liew, M.H. Zhang, et al. Development of cement-based strain sensor for health monitoring of ultra high strength concrete. Constr Build Mater, 2014; 65: 630-637. 